\title{
Sedimentation and polar order of active bottom-heavy particles
}

\author{
Katrin Wolffa , Aljoscha M. Hahn, and Holger Stark \\ Institut für Theoretische Physik, Technische Universität Berlin, Hardenbergstraße 36, 10623 Berlin, Germany
}

Received 14 December 2012

Published online: 25 April 2013

(C) The Author(s) 2013. This article is published with open access at Springerlink.com

\begin{abstract}
Self-propelled particles in an external gravitational field have been shown to display both an increased sedimentation length and polar order even without particle interactions. Here, we investigate self-propelled particles which additionally are bottom-heavy, that is they feel a torque aligning them to swim against the gravitational field. For bottom-heavy particles the gravitational field has the two opposite effects of i) sedimentation and ii) upward alignment of the particles' swimming direction. We perform a multipole expansion of the one-particle distribution of non-interacting particles with respect to orientation and derive expressions for sedimentation length and mean particle orientation which we check against Brownian Dynamics simulations. For large strength of gravity or small particle speeds and aligning torque, we observe sedimentation with increased sedimentation length compared with passive colloids but also active colloids without bottom-heaviness. Increasing, for example, swimming speed the sedimentation profile is inverted and the particles swim towards the top wall of the enclosing box. We find maximal orientational order at intermediate swimming speeds for both cases of particles with bottom-heaviness and those without. Ordering unsurprisingly is increased for the bottom-heavy particles, but this difference disappears at higher levels of activity and for very high activities ordering goes to zero in both cases.
\end{abstract}

\section{Introduction}

Actively moving particles, being intrinsically out of equilibrium, exhibit a number of novel phenomena including interesting collective behaviour. Local alignment leads to the formation of moving swarms $[1,2]$ or even turbulence [3] and hydrodynamic interactions may induce longrange order [4-6]. When particles slow down in the vicinity of others, this interplay between local particle density and speed leads to a motility-induced phase separation [7-12]. Autophoretic effects, where colloids move along (self-generated) gradients of fields such as concentration or temperature, give rise to collective behaviour reminiscent of chemotaxis in biological swimmers $[13,14]$.

But even in dilute suspensions, where particle interactions can be neglected, a number of interesting properties have been observed [15-18]. Activity changes the diffusive behaviour [19-22] and thus, for example, results in increased sedimentation lengths under gravitation [15-17]. While this effect can be mapped onto passive particles at higher temperatures, activity also aligns propulsion directions of particles against a gravitational field and orientational order can be found even without particle interactions $[17,18]$. This polar order is the combined ef-

\footnotetext{
a e-mail: katrin.wolff@tu-berlin.de
}

fect of self-propulsion and a non-constant density profile and appears without a gravitational torque aligning particles.

Here, we investigate non-interacting particles, which in addition to their activity exhibit bottom-heaviness, that is a particle's centre of mass is displaced from its geometric centre in such a way that the particle tends to "point upward" and thereby swims against an external gravitational field. For strong gravity, bottom-heaviness dominates particle orientation but in the colloidal regime, where thermal diffusion is not negligible, we observe a combination of activity-induced and bottom-heavy orientational order. Starting from the Smoluchowski equation for particle position and orientation, we derive an effective sedimentation process where activity and bottom-heaviness both enter into an increased effective diffusion constant and a reduced effective gravitation strength. We compare the analytically derived position density profile and orientational distribution function with results obtained from direct simulations of the equivalent Langevin equations. Furthermore, we identify a region in parameter space where the sedimentation profile is inverted and particles gather at the top wall of the enclosing box and determine density profiles at small to moderate Peclet numbers. This is the regime relevant for colloidal systems used, for example, in $[14,16]$. 


\section{Model system}

For bottom-heavy particles the centre of mass does not coincide with the geometric centre but is displaced toward the rear part of the particle, where front and rear are defined with respect to the swimming direction of the particle. The displacement is measured by the distance $r_{0}$. Gravity $\mathbf{g}$ acts on the centre of mass and the torque $\boldsymbol{\tau}=-m r_{0} \mathbf{p} \times \mathbf{g}$ tends to align the swimming direction $\mathbf{p}$ along $-\mathrm{g}$.

The resulting overdamped Langevin equations for particle position $\mathbf{r}$ and particle orientation $\mathbf{p}(|\mathbf{p}|=1)$ therefore read

$$
\begin{aligned}
& \dot{\mathbf{r}}=\mathbf{v}=v_{0} \mathbf{p}+\frac{m}{\gamma} \mathbf{g}+\sqrt{2 D} \boldsymbol{\xi}, \\
& \dot{\mathbf{p}}=\boldsymbol{\omega} \times \mathbf{p}=\frac{m r_{0}}{\gamma_{r}}(\mathbf{g} \times \mathbf{p}) \times \mathbf{p}+\sqrt{2 D_{r}} \boldsymbol{\zeta} \times \mathbf{p},
\end{aligned}
$$

in the Stratonovich interpretation. The deterministic drift terms $v_{0} \mathbf{p}$ and $m \mathbf{g} / \gamma$ give the swimming velocity of the active particle and the downward velocity due to gravity, respectively. The deterministic angular drift velocity is caused by the gravitational torque. While $m \mathbf{g} / \gamma$ actually requires the buoyancy mass $\Delta m=\left(\rho_{\text {colloid }}-\rho_{\text {water }}\right) R^{3}$ (density mismatch with surrounding fluid), the parameter in $m r_{0} / \gamma_{r}$ is indeed mass $m$. Here we assume that either $\Delta m \approx m$ or redefine the parameter $r_{0}$ as $r_{0} m / \Delta m$.

Both translational and rotational motion are subject to stochastic velocities $\sqrt{2 D} \boldsymbol{\xi}$ and $\sqrt{2 D_{r}} \boldsymbol{\zeta}$ where $\boldsymbol{\xi}$ and $\zeta$ each contain three independent Gaussian random variables with zero mean and unit variance. In this paper we assume that all noise is of thermal origin. So, the translational $(D)$ and rotational $\left(D_{r}\right)$ diffusion coefficients are connected with the respective friction coefficients via the Einstein relations $D=k_{B} T / \gamma$ and $D_{r}=k_{B} T / \gamma_{r}$. For spherical particles rotational and translational friction are related as $\gamma_{r}=4 / 3 R^{2} \gamma$ which, as the only source of randomness is thermal noise in the fluid, translates to $D_{r}=3 D /\left(4 R^{2}\right)$ for the diffusion coefficients.

We define a coordinate system by setting $\mathbf{g}=-g \mathbf{e}_{z}$ and non-dimensionalise spatial and temporal variables by setting $\mathbf{r}^{\prime}=\mathbf{r} / R$ and $t^{\prime}=t D / R^{2}$ and omitting the prime. This introduces the following dimensionless numbers, which we shall use as control parameters: the active Peclet number or simply activity, $\mathrm{Pe}=v_{0} R / D$, the gravitational Peclet number $\alpha=m g R /\left(k_{\mathrm{B}} T\right)$, which is the particle radius over sedimentation length of the passive system, and excentricity or bottom-heaviness $r_{0}^{\prime}=r_{0} / R$.

The equivalent Smoluchowski equation for the oneparticle density $\rho(\mathbf{r}, \mathbf{p}, t)$ depending on both position and orientation then is

$$
\begin{aligned}
\partial_{t} \rho= & -\nabla \cdot \mathbf{J}_{t}-\mathcal{R} \cdot \mathbf{J}_{r} \\
= & \nabla^{2} \rho+\left(\alpha \mathbf{e}_{z}-\operatorname{Pe} \mathbf{p}\right) \cdot \nabla \rho \\
& +\frac{3}{4}\left(\mathcal{R}^{2} \rho+\alpha r_{0} \mathcal{R} \cdot\left(\mathbf{e}_{z} \times \mathbf{p} \rho\right)\right),
\end{aligned}
$$

with $\mathcal{R}=\mathbf{p} \times \nabla_{\mathbf{p}}$ and the translational and rotational currents $\mathbf{J}_{t}=\left(\operatorname{Pep}-\alpha \mathbf{e}_{z}\right) \rho-\nabla \rho$ and $\mathbf{J}_{r}=-3 / 4 \alpha r_{0}\left(\mathbf{e}_{z} \times\right.$ p) $\rho-3 / 4 \mathcal{R} \rho$. At the system boundaries the translational current has to vanish.

\section{Multipole expansion}

In order to derive an approximative solution $\rho(\mathbf{r}, \mathbf{p}, t)$ to eq. (3) in a systematic way, we use the multipole expansion

$$
\begin{aligned}
\rho(\mathbf{r}, \mathbf{p}, t)= & \rho^{(0)}(\mathbf{r}, t)+\boldsymbol{\rho}^{(1)}(\mathbf{r}, t) \cdot \mathbf{p} \\
& +\boldsymbol{\rho}^{(2)}(\mathbf{r}, t):\left(\mathbf{p} \mathbf{p}-\frac{1}{3} \mathbb{I}\right)+\ldots,
\end{aligned}
$$

with coefficients $\rho^{(0)}(\mathbf{r}, t), \boldsymbol{\rho}^{(1)}(\mathbf{r}, t), \boldsymbol{\rho}^{(2)}(\mathbf{r}, t)$ depending on position and time but not on particle orientation. Since in our system of non-interacting sedimenting particles the only interesting dynamics takes place in $z$-direction, we assume that $\rho$ only depends on $z=r_{3}$ and $u=p_{3}=\cos \theta$. The multipole expansion for $\rho$ reduces to an expansion in Legendre polynomials

$$
\begin{aligned}
\rho(z, u, t)= & \rho^{(0)}(z, t)+\rho^{(1)}(z, t) u \\
& +\rho^{(2)}(z, t)\left(u^{2}-\frac{1}{3}\right)+\ldots,
\end{aligned}
$$

and the Smoluchowski equation (3) becomes

$$
\begin{aligned}
\partial_{t} \rho= & \partial_{z}^{2} \rho+(\alpha-\operatorname{Pe} u) \partial_{z} \rho \\
& +\frac{3}{4}\left(\partial_{u}\left(1-u^{2}\right) \partial_{u} \rho+\alpha r_{0} \partial_{u}\left(1-u^{2}\right) \rho\right) .
\end{aligned}
$$

The position-dependent density $\Omega(z, t)=\int_{-1}^{1} \rho(z, u, t) \mathrm{d} u$, which is an easy observable in experiments, is related to the zeroth-order expansion coefficient $\rho^{(0)}(z, t)$ simply as $\Omega(z, t)=2 \rho^{(0)}(z, t)$. We begin with deriving an effective drift-diffusion process for density $\Omega(z, t)$ valid on time scales larger than the orientational decorrelation time $\propto D_{r}^{-1}$. It is of the form

$$
\partial_{t} \Omega=-\partial_{z} J_{\Omega}
$$

where $J_{\Omega}=\alpha_{\text {eff }} \Omega-D_{\text {eff }} \partial_{z} \Omega$ is the effective density current with the drift and diffusive part.

We now derive equations for coefficients $\rho^{(0)}, \rho^{(1)}$ and $\rho^{(2)}$ from eq. (6) making use of the orthogonality of Legendre polynomials. We first integrate eq. (6) over $u$ to obtain

$$
\partial_{t} \rho^{(0)}=\partial_{z}^{2} \rho^{(0)}+\partial_{z}\left(\alpha \rho^{(0)}-\frac{\mathrm{Pe}}{3} \rho^{(1)}\right) .
$$

Multiplying eq. (6) by $u$ and then integrating over $u$ yields the equation for $\rho^{(1)}$

$$
\begin{aligned}
\partial_{t} \rho^{(1)}= & \partial_{z}^{2} \rho^{(1)}+\alpha \partial_{z} \rho^{(1)}-\operatorname{Pe} \partial_{z}\left(\frac{4}{15} \rho^{(2)}+\rho^{(0)}\right) \\
& -\frac{3}{2} \rho^{(1)}+\alpha r_{0}\left(\frac{3}{2} \rho^{(0)}-\frac{1}{5} \rho^{(2)}\right)
\end{aligned}
$$

The same procedure for $u^{2}-1 / 3$ gives the next order

$$
\begin{aligned}
\partial_{t} \rho^{(2)}= & \partial_{z}^{2} \rho^{(2)}+\alpha \partial_{z} \rho^{(2)}-\operatorname{Pe} \partial_{z}\left(\frac{9}{35} \rho^{(3)}+\rho^{(1)}\right) \\
& -\frac{9}{2} \rho^{(2)}+\alpha r_{0}\left(\frac{9}{4} \rho^{(1)}-\frac{27}{70} \rho^{(3)}\right) .
\end{aligned}
$$


So, each equation couples $\rho^{(i)}$ to the next higher-order $\rho^{(i+1)}$. Equations (9) and (10) describe massive modes with $\partial_{t} \rho^{(i)} \propto \rho^{(i)}, i=1,2$, which relax faster than the slow mode $\partial_{t} \rho^{(0)} \propto \partial_{z} \rho^{(0)}$ from eq. (8). We therefore omit the time derivatives in eqs. (9) and (10) while keeping the time dependence in eq. (8) (cf. refs. [11,13]). Furthermore, we want to keep eq. (7) at the drift-diffusion level with at most second-order derivatives and therefore drop secondorder derivatives in eq. (9) and second- and first-order derivatives in eq. (10). We are still left with an equation for $\rho^{(2)}$ that couples it to the next higher-order $\rho^{(3)}$. This coupling is due to the bottom-heaviness of particles as would be the case with any rotational drift term. To close the relation, we assume $\rho^{(3)} \ll \rho^{(1)}$ and arrive at

$$
\rho^{(2)}=\frac{\alpha r_{0}}{2} \rho^{(1)} \text {. }
$$

We plug this result into eq. (9) and obtain an equation in $\rho^{(1)}$. Taking its derivative with respect to $z$ and neglecting second-order derivatives gives $\partial_{z} \rho^{(1)} \propto \partial_{z} \rho^{(0)}$, which we re-insert into the equation for $\rho^{(1)}$ to obtain

$\rho^{(1)}=\frac{2}{3+\left(\alpha r_{0}\right)^{2} / 5}\left((3 A-\mathrm{Pe}) \partial_{z} \rho^{(0)}+\frac{3}{2} \alpha r_{0} \rho^{(0)}\right)$,

with $A=\left(\alpha^{2} r_{0}-2\left(\alpha r_{0}\right)^{2} \mathrm{Pe} / 15\right) /\left(3+\left(\alpha r_{0}\right)^{2} / 5\right)$. Using this result in eq. (8), finally gives

$$
\partial_{t} \rho^{(0)}=D_{\mathrm{eff}} \partial_{z}^{2} \rho^{(0)}-\alpha_{\mathrm{eff}} \partial_{z} \rho^{(0)},
$$

with effective diffusion coefficient

$$
D_{\text {eff }}=1+\frac{2 \mathrm{Pe}}{3+\left(\alpha r_{0}\right)^{2} / 5}\left(\frac{\mathrm{Pe}}{3}-A\right)
$$

and effective gravity

$$
\alpha_{\mathrm{eff}}=\alpha\left(1-\frac{r_{0} \mathrm{Pe}}{3+\left(\alpha r_{0}\right)^{2} / 5}\right) .
$$

We have thus identified the coefficients for the driftdiffusion equation for $\Omega(z, t)=2 \rho^{(0)}(z, t)$ in eq. (7).

The steady-state solution for $\Omega(z)$ is an exponential profile with inverse sedimentation length $\alpha_{\text {eff }} / D_{\text {eff }}$, $\Omega \propto \exp \left(-\alpha_{\text {eff }} z / D_{\text {eff }}\right)$. We are now in a position to judge when the approximation to neglect derivatives of $\rho^{(i)}$, which we made in the derivation, is justified. As $\rho^{(i)} \propto$ $\exp \left(-\alpha_{\text {eff }} z / D_{\text {eff }}\right)$ for $i=0,1,2$ (eqs. (11), (12), (13)), this approximation is possible when the absolute value of the reduced inverse sedimentation length $\left|\alpha_{\text {eff }} / D_{\text {eff }}\right|<1$. For $\alpha \approx 1$ passive colloids have a sedimentation length equal to the particle radius $R$ meaning that particles accumulate at the bottom wall. Then, $\mathrm{Pe} \propto v_{0}$ and thereby $D_{\text {eff }}$ have to be sufficiently large, so that active particles can leave this densely packed colloidal phase.

Within the regime of our approximations, we see that $D_{\text {eff }}$ increases quadratically with $\mathrm{Pe}$ in accordance with the well-known result for the diffusion of (non-bottomheavy) active particles $[19,20]$. The aligning torque $\left(\alpha r_{0}\right)$ counteracts this trend as it tends to rectify particle swimming directions and thereby decrease the randomness in the active motion. For very large $\alpha r_{0}$ the diffusion coefficient reverts to the passive case of purely thermal noise as swimming becomes fully deterministic. The effective gravitational strength $\alpha_{\text {eff }}$ goes roughly linearly in $\alpha$. Both bottom-heaviness $\left(r_{0}\right)$ and activity $(\mathrm{Pe})$ work against gravity by causing the particles to swim upward. For $\alpha_{\text {eff }}<0$, activity together with bottom-heaviness overcomes gravity and particles swim towards the top resulting in inverted density profiles. For $\alpha_{\text {eff }}=0$ the two effects balance and the density profile becomes uniform or constant in $z$ (far from the confining walls). The limits of our approximation again become visible for very large $r_{0}$ where eq. (15) predicts $\alpha_{\text {eff }}$ to tend to $\alpha$ instead of $\alpha-$ Pe.

The swimming speed necessary to cancel sedimentation and to obtain a uniform density profile can also be calculated independently of the multipole expansion. A solution of eq. (6) that is constant in $z$ corresponds to the Boltzmann distribution of the particle orientation under the aligning torque, which gives $\rho(z, u) \propto \exp \left(\alpha r_{0} u\right)$, also known as Mises-Fisher distribution. Since the bounding walls enforce that the average translational drift current along the $z$-direction is zero, one has $0=\operatorname{Pe}\langle u\rangle-\alpha$. Calculating $\langle u\rangle$ from the Boltzmann distribution, we obtain

$$
\mathrm{Pe}^{*}=\frac{\alpha}{\operatorname{coth}\left(\alpha r_{0}\right)-\frac{1}{\alpha r_{0}}}
$$

for the activity $\mathrm{Pe}^{*}$ which cancels gravity so that a uniform density profile occurs. The approximate result for $\mathrm{Pe}^{*}$ found from eq. (15) by setting $\alpha_{\mathrm{eff}}=0$ amounts to

$$
\mathrm{Pe}^{*} \approx \frac{3}{r_{0}}+\frac{\alpha^{2} r_{0}}{5}
$$

It corresponds to the first two terms of the Laurent series of eq. (16) in $\alpha r_{0}$.

\section{Sedimentation length and orientational order}

We solved the Langevin equations (1) and (2) for the system of bottom-heavy active particles by performing Brownian Dynamics simulations. We chose a cubic simulation box of size $L^{3}$ with $L=60$ (in units of particle radii) with hard walls at $z=0$ and $z=L$ and periodic boundary conditions in $x$ - and $y$-direction. For low levels of activity (see fig. $1(\mathrm{a})$ for $\mathrm{Pe}=3$ ) the sole effect of bottom-heaviness is an increase in sedimentation length, while at higher activity, $\mathrm{Pe}=8$, bottom-heavy particles overcome gravity and produce inverted sedimentation profiles with particles swimming towards the top of the simulation box. The constant profile is realised for $\mathrm{Pe}=\mathrm{Pe}^{*} \approx 6.1$. All three cases are captured very well by $\Omega(z) \propto \exp \left(-\alpha_{\text {eff }} / D_{\text {eff }}\right)$ as can be seen in the comparison of simulations (dots) and theory (dashed lines) in fig. 1(a). The only significant deviations between the two appear at the hard walls in $z$ which have been neglected in the theory. In the simulations, the boundary conditions in $z$ give rise to an accumulation of particles swimming against the wall and thus an increase in density next to the wall. The net orientation at the wall 
(a)

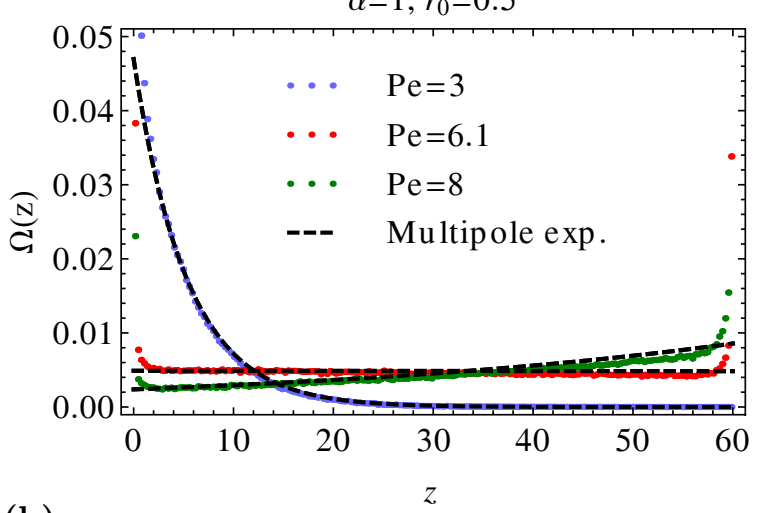

(b)

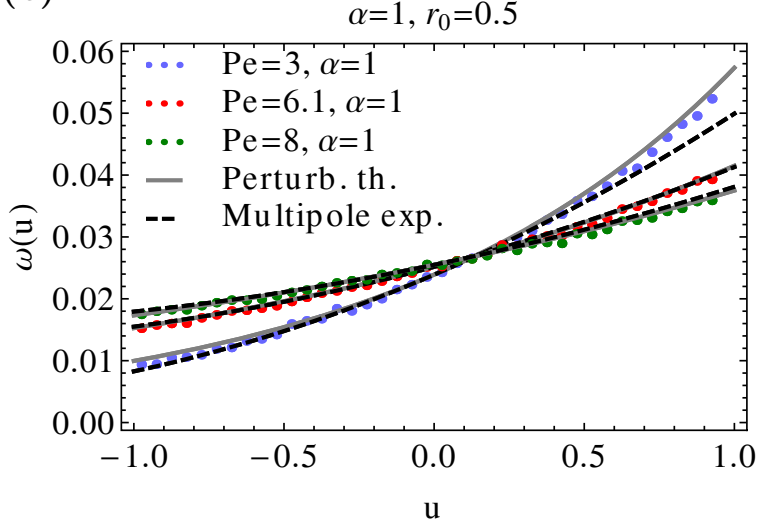

Fig. 1. (Colour online) (a) Spatial density profile $\Omega(z)$ and (b) orientational distribution $\omega(u)$ (within the bulk). Numerical data (dots) are computed from Brownian Dynamics simulations of eqs. (1) and (2). Gravitational strength $\alpha=1$ and bottom-heaviness $r_{0}=0.5$ are kept constant, activity is $\mathrm{Pe}=3$ (blue dots), $\mathrm{Pe}=6.1 \approx \mathrm{Pe}^{*}$ (red dots) and $\mathrm{Pe}=8$ (green dots).

also differs from the bulk (not shown). This effect has been discussed in [17] and accumulation of colloids next to the walls has also been observed in experiments [16].

The stationary orientational distribution function $\omega(u \mid z)=\rho(z, u) / \Omega(z)$ is found to be independent of position $z$ except in the vicinity of the bounding walls. We therefore write $\omega(u \mid z)=\omega(u)$ for short. The orientational distribution also depends on the particles' activity as can be seen from fig. 1(b). This ordering is due to two effects: Firstly, the bottom-heaviness simply aligns particles against the vertical while the second effect due to activity works more subtly and in conjunction with the spatial density profile as shown in ref. [17]. An excess of particles at position $z-\Delta z$ will mean that more particles reach position $z$ swimming upwards from below than swimming downwards from above and particles at $z$ will predominantly point upwards. At low activity, $\mathrm{Pe}=3$, the sedimentation profile is more pronounced and it produces alignment in the same direction as bottom-heaviness. In contrast, an inverted profile such as for $\mathrm{Pe}=8$ works in the opposite direction; it reduces orientational order and results in a flatter distribution, see fig. 1(b). The orientational distribution $\omega(u)$ can also be derived from the mul- tipole expansion eq. (5). After insertion of $\rho^{(i)}, i=0,1,2$ we find

$$
\rho(u, z)=\Omega(z) \omega(u),
$$

with

$$
\begin{aligned}
\omega(u) \propto & 1+\frac{1}{3+\left(\alpha r_{0}\right)^{2} / 5}\left(\frac{\alpha_{\mathrm{eff}}}{D_{\mathrm{eff}}}(\mathrm{Pe}-3 A)+\frac{3}{2} \alpha r_{0}\right) \\
& \times\left[2 u+\alpha r_{0}\left(u^{2}-\frac{1}{3}\right)\right] .
\end{aligned}
$$

As we only performed the expansion up to second order, $\omega(u)$ is quadratic in $u$ by construction. A better fit of the data, but a less systematic result, can be found from a perturbation ansatz in $\alpha, \omega(u)=\exp \left(\alpha f_{1}(u)+\alpha^{2} f_{2}(u)\right)$, where $f_{1}(u)$ and $f_{2}(u)$ can be determined from inserting eq. (18) into eq. (6). Both results are plotted against simulation data in fig. $1(\mathrm{~b})$.

We find that sedimentation lengths increase with activity Pe for both bottom-heavy and non-bottom-heavy particles. However, the increase for bottom-heavy particles is stronger as fig. 2(a) demonstrates where we plot inverse sedimentation length $\alpha_{\text {eff }} / D_{\text {eff }}$ over Pe. For large Pe both curves converge to $\alpha_{\text {eff }} / D_{\text {eff }}=0$ (infinite sedimentation length) as the effective diffusion diverges quadratically in $\mathrm{Pe}$, that is the contribution of activity to random motion increases faster with $\mathrm{Pe}$ than the contribution to directed motion. However, the curve for bottom-heavy particles does so from below as for large Pe upswimming wins over sedimentation and the drift term becomes negative, $\alpha_{\text {eff }}<0$. This regime of inverted profiles is visible even better in fig. 2(b), where inverse sedimentation length is plotted against gravitational strength $\alpha$. For intermediate values of $\alpha$, bottom-heavy particles are oriented upwards but gravity is not yet strong enough to pull particles down and we find maximally inverted profiles for $\alpha \approx 3$ (dashed blue curve). For non-bottom-heavy particles (solid red curve) the inverse sedimentation length increases linearly for small $\alpha$, in agreement with ref. [17]. Deviations between simulation and theory appear once sedimentation lengths become too small, as discussed before. Parameter ranges relevant for active colloidal systems in ref. [16] are $\alpha=0.1$ and $\mathrm{Pe}=0$ to $\mathrm{Pe}=6$ but larger values could be realised by increasing particle radii since $\alpha \propto R^{4}$ and $\mathrm{Pe} \propto R$. The region of inverted sedimentation for bottomheavy particles is especially interesting as tuning of $\alpha$ in a centrifuge might be used to separate bottom-heavy from non-bottom-heavy colloids.

As has been mentioned above, the orientational distribution changes with activity $\mathrm{Pe}$ and becomes flatter for large values. Indeed, we find a maximum in mean particle orientation $\langle u\rangle=\Omega(z)^{-1} \int_{-1}^{1} \rho(u, z) u \mathrm{~d} u$ for intermediate values of activity. From $\langle u\rangle=\rho^{(1)} /\left(3 \rho^{(0)}\right)$ we then find

$$
\langle u\rangle=\frac{2}{3+\left(\alpha r_{0}\right)^{2} / 5}\left(\frac{\alpha_{\mathrm{eff}}}{D_{\mathrm{eff}}}\left(\frac{\mathrm{Pe}}{3}-A\right)+\frac{1}{2} \alpha r_{0}\right)
$$

which at large Pe can be roughly viewed as an activityinduced part $\propto \alpha_{\text {eff }} / D_{\text {eff }}$ (although depending on the spatial profile which is changed by the presence of bottom- 
(a)

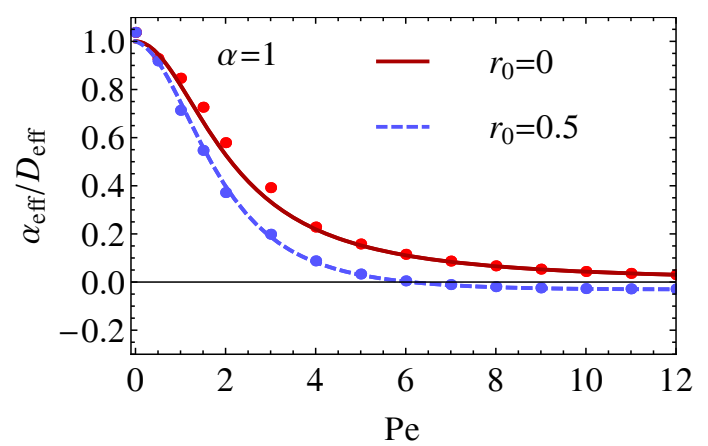

(b)

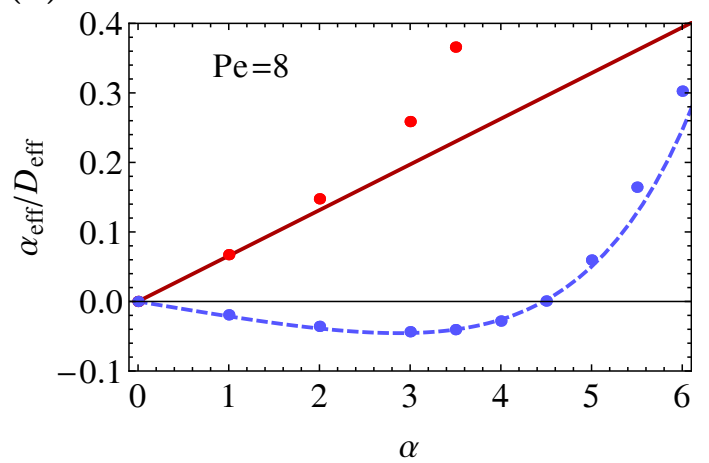

Fig. 2. (Colour online) Inverse sedimentation length $\alpha_{\text {eff }} / D_{\text {eff }}$ (a) over activity $\mathrm{Pe}$ at constant $\alpha=1$ and (b) over gravitational strength $\alpha$ at constant $\mathrm{Pe}=8$. Lines are theoretical predictions, dots are simulation results. Curves for non-bottomheavy particles $\left(r_{0}=0\right)$ are shown in solid red, curves for bottom-heavy particles $\left(r_{0}=0.5\right)$ are dashed blue.

heaviness) and the direct contribution due to bottomheaviness. In fig. 3(a) for fixed $\alpha=1$, polar order is maximal for $\mathrm{Pe} \approx 2$ for non-bottom-heavy particles and for slightly lower values for bottom-heavy particles. Unsurprisingly, ordering is stronger for bottom-heavy particles as bottom-heaviness itself directly contributes to particle alignment. For very large activities, however, $\langle u\rangle$ tends to zero in both cases. For non-bottom-heavy particles this is simply explained by $\alpha_{\text {eff }} / D_{\text {eff }}$ going to zero as $\mathrm{Pe}^{-2}$. Physically, this means the spatial density profile $\Omega(z)$ becomes constant and activity-induced order $\propto \operatorname{Pe} \alpha_{\text {eff }} / D_{\text {eff }}$ vanishes. For bottom-heavy particles $\alpha_{\text {eff }} / D_{\text {eff }}$ goes to zero only as $\mathrm{Pe}^{-1}$ and activity-induced order becomes constant and cancels the bottom-heavy contribution.

Ordering increases linearly with $\alpha$ for small $\alpha$, see fig. 3(b), but flattens out for increasing values. For bottom-heavy particles the effect from increasing $\alpha$ is twofold as it enhances both the passive alignment in an external potential and activity-induced alignment by sharpening the sedimentation profile. Mean orientation therefore increases with $\alpha$ faster for bottom-heavy particles than it does for non-bottom-heavy particles although at the relatively large activity of $\mathrm{Pe}=8$ the curves indicate that the main effect for orientational order comes from the sedimentation profile. (a)

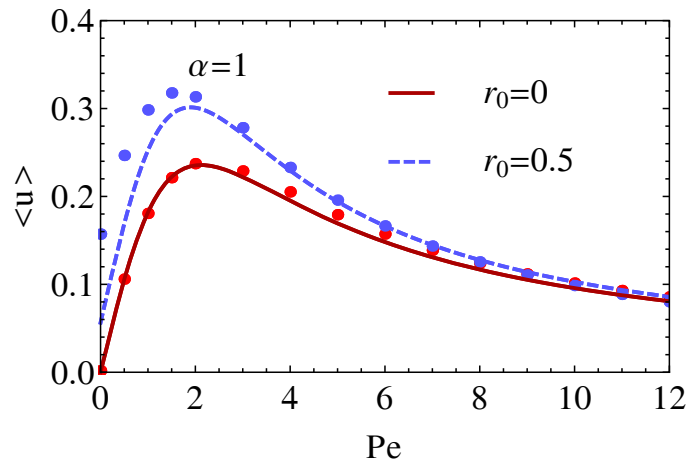

(b)

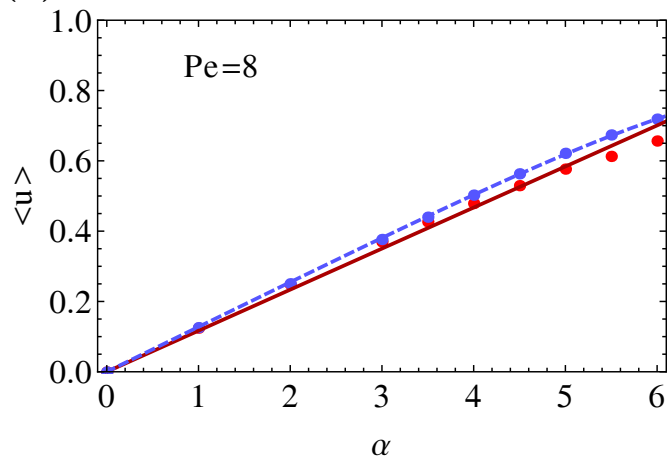

Fig. 3. (Colour online) Mean orientation $\langle u\rangle$ (a) over activity Pe at constant $\alpha=1$ and (b) over gravitational strength $\alpha$ at constant $\mathrm{Pe}=8$. Lines are theoretical predictions, dots are simulation results. Curves for non-bottom-heavy particles $\left(r_{0}=0\right)$ are shown in solid red, curves for bottom-heavy particles $\left(r_{0}=0.5\right)$ are dashed blue.

\section{Conclusion}

To conclude, we performed a multipole expansion up to second order in orientation for the one-particle density of active bottom-heavy particles and determined a solution of the Smoluchowski equation in dilute suspensions which is prerequisite to the study of interacting particles. This approximation is useful in the colloidal regime where active motion and gravity are not too dominant compared with thermal diffusion and the regime explored here is experimentally accessible, e.g., by chemically powered Janus particles $[14,16]$. Comparing orientational order in bottom-heavy and non-bottom-heavy particles, we elucidated the influence of both activity (cf. ref. [17]) and of bottom-heaviness.

We derived analytical expressions for the sedimentation length of bottom-heavy particles and discussed the two regimes of increased sedimentation lengths [15-17] and of inverted sedimentation profiles. For fixed gravitational strength $\alpha$ or fixed activity Pe, we find optimal values for the other parameter to maximise inversion. It is this density inversion and its instability under density fluctuations that, in the case of interacting particles, gives rise to pattern formation by bioconvection [23-25] (albeit in a parameter regime of large Peclet numbers where 
thermal diffusion is small compared with active motion). We are currently working on how this instability is induced by hydrodynamic interactions between individual swimmers. Recently, it was demonstrated that in case of two Volvox algae hydrodynamic coupling leads to intricate bound states [26]. Finally, we note that alignment of particles can also be achieved by mechanisms other than bottom-heaviness. For example, for superparamagnetic colloids [27] or magnetic Janus spheres [28] alignment is easily tuned by an external magnetic field whereas for biological swimmers chemotaxis or phototaxis result in similar phenomena [29].

We gratefully acknowledge financial support by the Deutsche Forschungsgemeinschaft through the Research Training Group GRK1558.

Open Access This is an open access article distributed under the terms of the Creative Commons Attribution License (http://creativecommons.org/licenses/by/3.0), which permits unrestricted use, distribution, and reproduction in any medium, provided the original work is properly cited.

\section{References}

1. T. Vicsek, A. Czirók, E. Ben-Jacob, I. Cohen, O. Shochet, Phys. Rev. Lett. 75, 1226 (1995).

2. F. Ginelli, F. Peruani, M. Bär, H. Chaté, Phys. Rev. Lett. 104, 184502 (2010).

3. H.H. Wensink, J. Dunkel, S. Heidenreich, K. Drescher, R.E. Goldstein, H. Löwen, J.M. Yeomans, Proc. Natl. Acad. Sci. U.S.A. 109, 14308 (2012).

4. R.A. Simha, S. Ramaswamy, Phys. Rev. Lett. 89, 058101 (2002).

5. D. Saintillan, M.J. Shelley, Phys. Rev. Lett. 100, 178103 (2008).
6. A. Baskaran, M.C. Marchetti, Proc. Natl. Acad. Sci. U.S.A. 106, 15567 (2009).

7. J. Tailleur, M.E. Cates, Phys. Rev. Lett. 100, 218103 (2008).

8. J. Bialké, T. Speck, H. Löwen, Phys. Rev. Lett. 108, 168301 (2012).

9. F.D.C. Farrell, M.C. Marchetti, D. Marenduzzo, J. Tailleur, Phys. Rev. Lett. 108, 248101 (2012).

10. Y. Fily, M.C. Marchetti, Phys. Rev. Lett. 108, 235702 (2012).

11. M.E. Cates, J. Tailleur, EPL 101, 20010 (2013).

12. G.S. Redner, M.F. Hagan, A. Baskaran, Phys. Rev. Lett. 110, 055701 (2013).

13. R. Golestanian, Phys. Rev. Lett. 108, 038303 (2012).

14. I. Theurkauff, C. Cottin-Bizonne, J. Palacci, C. Ybert, L. Bocquet, Phys. Rev. Lett. 108, 268303 (2012).

15. J. Tailleur, M.E. Cates, EPL 86, 60002 (2009).

16. J. Palacci, C. Cottin-Bizonne, C. Ybert, L. Bocquet, Phys. Rev. Lett. 105, 088304 (2010).

17. M. Enculescu, H. Stark, Phys. Rev. Lett. 107, 058301 (2011).

18. A. Pototsky, H. Stark, EPL 98, 50004 (2012).

19. H.C. Berg, Random Walks in Biology (Princeton University Press, 1993).

20. J.R. Howse, R.A.L. Jones, A.J. Ryan, T. Gough, R. Vafabakhsh, R. Golestanian, Phys. Rev. Lett. 99, 048102 (2007).

21. R. Golestanian, Phys. Rev. Lett. 102, 188305 (2009).

22. P. Romanczuk, M. Bär, W. Ebeling, B. Lindner, L. Schimansky-Geier, Eur. Phys. J. ST 202, 1 (2012).

23. S. Childress, M. Levandowsky, E.A. Spiegel, J. Fluid Mech. 69, 591 (1975).

24. T.J. Pedley, J.O. Kessler, J. Fluid Mech. 212, 155 (1990).

25. M.A. Bees, N.A. Hill, Phys. Fluids 10, 1864 (1998).

26. K. Drescher, K.C. Leptos, I. Tuval, T. Ishikawa, T.J. Pedley, R.E. Goldstein, Phys. Rev. Lett. 102, 168101 (2009).

27. F. Ebert, P. Dillmann, G. Maret, P. Keim, Rev. Sci. Instrum. 80, 083902 (2009).

28. J. Yan, M. Bloom, S.C. Bae, E. Luijten, S. Granick, Nature 491, 578 (2012).

29. C.R. Williams, M.A. Bees, J. Exp. Biol. 214, 2398 (2011). 